\title{
Improved Design Scheme for the Automatic Monitoring System of Settlement
}

\author{
Haobo Dong, Lan Tang* and Ping Wu \\ Xihua University, China \\ *Corresponding author
}

\begin{abstract}
This paper introduces the principle of the automated subsidence monitoring system, and pointed out that it is not ideal for the identification of the interference caused by the interference of the external environment and the wide range of environmental temperature range. And then put forward the solution, adding vibration sensor and temperature sensor two modules. It realizes the vibration interference judgment before sampling and the temperature compensation after sampling. Finally, the reliability of the improved system is verified by experiments. The results show that the vibration module can eliminate most of the unusual points, and the temperature module greatly improves the stability of the data, and the standard deviation is reduced more than 10 times. Therefore, the improved settlement monitoring system has high monitoring accuracy and practical value.
\end{abstract}

Keywords-monitoring system of settlement; interference judgment; temperature compensation; monitoring precision

\section{INTRODUCTION}

With the rapid development of domestic high speed railways and roads, there are a lot of railway and highway every year. Operating lines of settlement of abnormal changes of railway operation management and public works maintenance bring severe challenges, to ensure the safety of the high-speed railway running peace shun, embankment settlement measurement and monitoring has become a railway construction and operation period of a key technical problem. At home and abroad about the settlement deformation monitoring method is observation pile, the settlement plate, single point settlement instrument, etc., there are a lot of disadvantages, such as only to complete the automation of data collection and transmission, and to affect the monitoring accuracy of external disturbance, (such as the train vibration, construction interference, human touch, etc.) and the external environment temperature did not recognize or blocked. So in order to promote the accuracy of subsidence monitoring system, ensure the normal operation of railway, needs some improvements to the settlement of the existing monitoring system.

\section{Settlement Of Automatic Monitoring System WORKING PRINCIPLE AND COMPOSITION}

\section{A. Working Principle}

The static level automatic monitoring system based on the principle of fluid connectors, through the different measuring point of the static level measurement of liquid level height difference of datum and were relatively subsidence monitoring. In practice, related to the static level by infusion tube connected to each other, one of them or part of the static level as a reference point, other static level as the observation point, synergy complete a settlement measurement of the measuring point.datum mark and reference surface (water tank liquid level) the height difference between the Ho, observation point and reference between liquid level height is $\mathrm{Hi}$, Hi respectively measured values with $\mathrm{Ho}, \mathrm{Hi}-\mathrm{Ho}$ has a value of observation point relative to the reference point the height of the delta. When the height of the observation point changes, the delta of the measured values and the corresponding change, two delta value is the difference between the settlement value ${ }^{[1]}$.The measurement principle is shown in Figure 1.

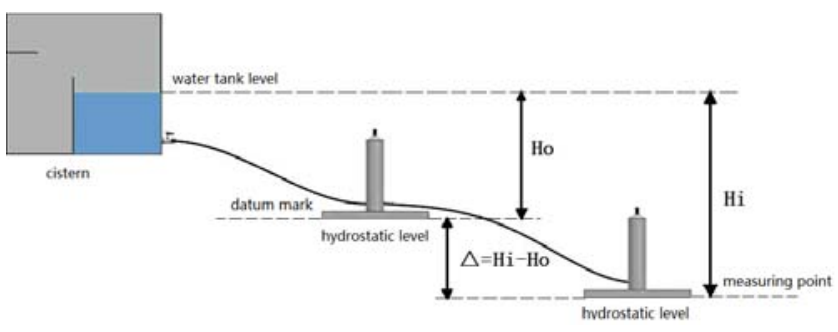

FIGURE I. THE STATIC LEVEL MEASURING PRINCIPLE DIAGRAM

\section{B. System Composition Distributed}

System architecture, the system adopts advanced by the field monitoring equipment, control unit, the wireless network module and a central server system.

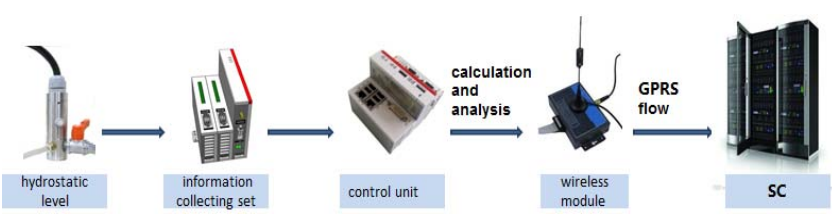

FIGURE II. SUBSIDENCE AUTOMATIC MONITORING SYSTEM STRUCTURE DIAGRAM

Select the appropriate monitoring equipment to improve the reliability and accuracy of the whole monitoring system, and for the next step of data analysis, forecasting, etc. To provide scientific and reliable data. The static level has high resolution of $0.01 \mathrm{~mm}$ and $0.1 \mathrm{~mm}$ high precision, higher stability and reliability of measurement, and the working conditions at $25{ }^{\circ} \mathrm{C}$ $\sim+80{ }^{\circ} \mathrm{C}$, etc, can satisfy the requirement of monitoring equipment. 


\section{Sedimentation Simulation Experiment Device}

The settlement deformation of simulated test by telex servo control system, control the motor to drive the test rig on top of the test stand bracket to move up and down. The simulation system can sat-isfy the requirement of low rate of settlement deformation, besides can simulate conventional settle-ment deformation, can be simulated with external interference sources under the settlement, and foot long time settling deformation simulation requirements.

Using the thermostat temperature adjustment function to simulate the temperature change of the external environment, coupled with the settlement of the above simulation test rig simulation in the outside temperature range is larger under the interference of ground subsidence. Sedimentation simulation test bench and incubator simulation diagram, as shown in Figure 3.

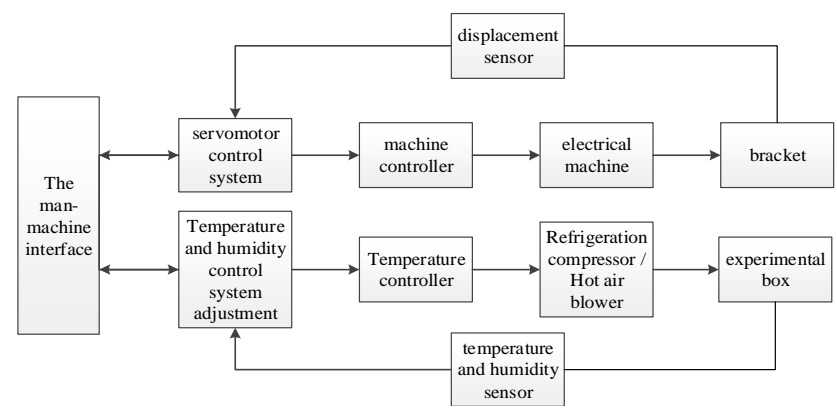

FIGURE III. SEDIMENTATION SIMULATION TEST BENCH AND SIMULATION FRAME OF INCUBATOR

\section{IMPROVE THE MONITORING ACCURACY OF SYSTEM SOLUTIONS}

\section{A. Filtering Fusion Algorithm}

Filtering fusion algorithm is to separate sensor data to carry on the synthesis, it is the basis of the data, that is, any sensor is as a module of the fusion system. A sensor from the limitation of single sensor, forming a very loose structure, a sensor system "into", or "pulling" from the system, not affect the normal work of the whole system. Even if a certain sensor "sick", nor would spread disease to other sensors and the whole system. Of each sensor in the landslide monitoring system, the displacement is relevant, according to the feedback structure of information fusion.

Have feedback information distributed fusion structure, not only before each sensor fusion filtering, and by the fusion center to each sensor has a feedback channel, the need for secondary filter. Obviously, this is helpful to improve each sensor state estimation and prediction accuracy. Compared with no feedback distributed structure, of course, it increases the traffic, pay attention to the participation of the correlation between the amount of calculation.

\section{B. In View of the outside Temperature Change Interference Solution}

Using subsidence simulation test bench and constant temperature box in the temperature range larger external environment under the interference of ground subsidence. Set the parameters as shown below:

Constant temperature box temperature range for: $-20{ }^{\circ} \mathrm{C}$ to $40{ }^{\circ} \mathrm{C}$; Variable temperature way is: the linear change, rate is: $1.25{ }^{\circ} \mathrm{C} / 30 \mathrm{~min}$; Frequency of the collection device to upload: $1 / 60 \mathrm{~Hz}$; Test time is 3.5 days, namely the upload times to 5040 times.

Set up the two experiments, experiment in each group the static level of the four with a temperature sensor modules, a total of eight sets of static level.

1 the static level, for example, according to the sample value and the actual value of the error, fitting the curve of the Figure 4, according to the curve fitting out, for temperature compensation.
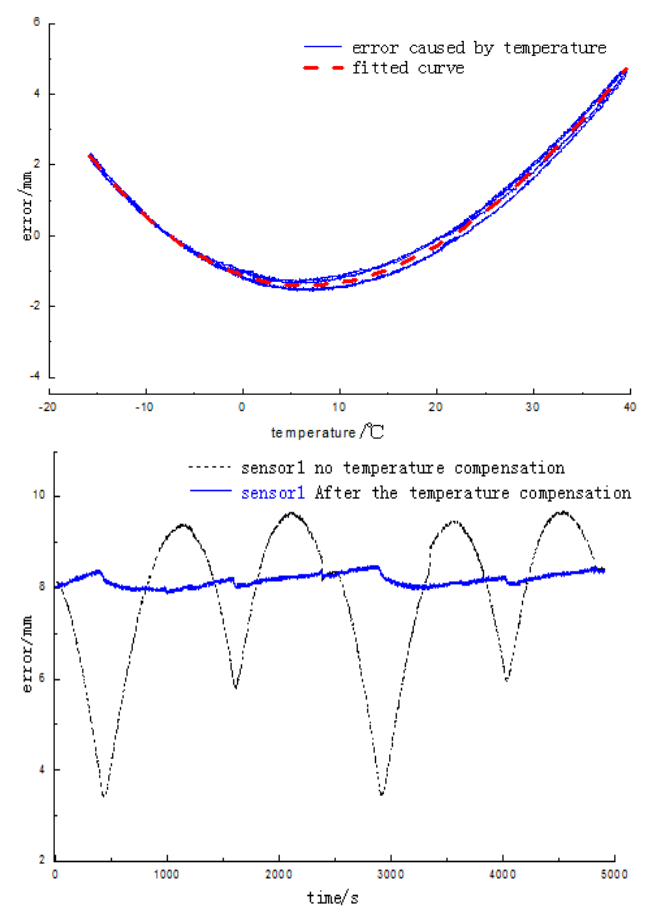

FIGURE IV. THE STATIC LEVEL FIT CURVE OF TEMPERATURE COMPENSATION AND 1 STATIC LEVEL BEFORE AND AFTER REMOVING CONTRAST FIGURE

Fitting curve obtained from the Fig. 4 a three order curve formula is as follows:

$$
y=-0.0000334 x^{\wedge} 3+0.00734 x^{\wedge} 2-0.0911 x-1.11714
$$

According to this formula can achieve sampling after temperature compensation. 1 the static level, for example, do not make removing and contrast samples after removing, as shown in Figure 4.

After the completion of the two experiments, eight sets of the static level before and after the temperature compensation of the experimental results are shown in table below. 
TABLE I. THE STATIC LEVEL BEFORE AND AFTER REMOVING THE EXPERIMENTAL RESULTS

\begin{tabular}{cccccc}
\hline & \multicolumn{2}{c}{ Before } & \multicolumn{2}{c}{ after } & after \\
$\begin{array}{c}\text { hydrostatic } \\
\text { level }\end{array}$ & $\begin{array}{c}\text { before } \\
\text { TC }\end{array}$ & $\begin{array}{c}\text { TC } \\
\text { standard } \\
\text { label }\end{array}$ & $\begin{array}{c}\text { TC } \\
\text { mean } \\
\text { mean value }\end{array}$ & $\begin{array}{c}\text { TC } \\
\text { standard } \\
\text { deviation }\end{array}$ \\
\hline No.1 & 7.755 & 1.665 & 8.153 & 0.134 & \\
No.2 & 6.225 & 1.382 & 6.935 & 0.122 & \\
No.3 & 6.855 & 1.529 & 7.730 & 0.173 & \\
No.4 & 6.324 & 1.114 & 6.970 & 0.114 & \\
No.5 & 8.348 & 2.334 & 7.921 & 0.155 & \\
No.6 & 7.111 & 1.513 & 8.227 & 0.130 & \\
No.7 & 9.086 & 2.534 & 8.435 & 0.173 & \\
No.8 & 6.970 & 1.425 & 7.839 & 0.141 & \\
\hline
\end{tabular}

Label: TC means temperature compensation.

According to the experimental results of table 1 can be seen that, after temperature compensation, the static level of sampling accuracy obviously than before compensation of high precision.

\section{In View of the External Disturbance Solution}

An ever-present precisely monitoring equipment of external interference, to train through construction, vibration, the vibration of the small to barely visible. Obviously, the vibration affected the accuracy of monitoring equipment acquisition are those of natural vibration. Based on the objective facts, join vibration sensors on monitoring equipment can solve this problem very well.

Experiment preparation: first, determine whether there are external vibration interference logic flow diagram as shown below.

The judgment principle for limiting filtering method (also known as judgment filtering method) program. Based on experience judgement, determine the sampling allows the maximum vibration (to A). When every time A new value detected to determine: if the vibration samples values less than or equal to $\mathrm{A}$, the settlement of the monitoring equipment samples values effectively; Sampling value is greater than A, if the vibration is the settlement of the monitoring equipment, sampling value is invalid. Repeat the above judgment until the upload data $\mathrm{N}$.

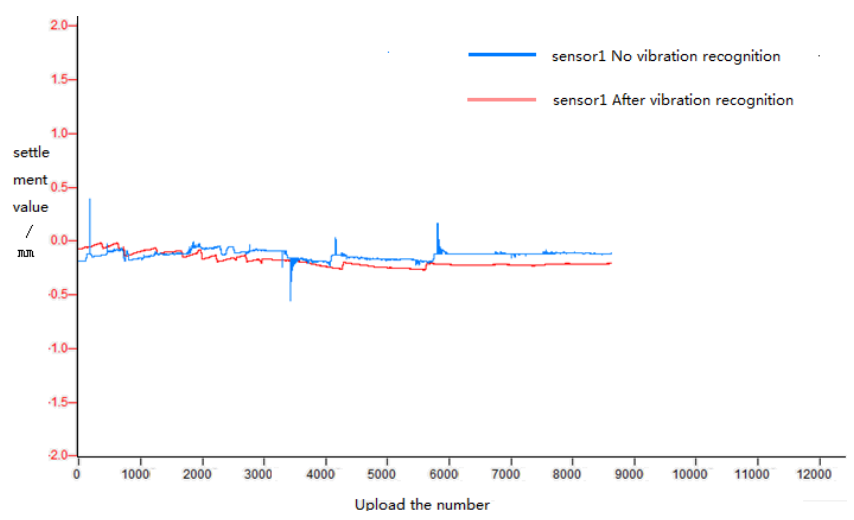

FIGURE V. A STATIC LEVEL BEFORE AND AFTER THE VIBRATION IDENTIFICATION CONTRAST FIGURE
Experimental steps: set up the two experiments, the first set of experiments, set up four units don't provide the static level of vibration sensor module, a second set of experiments set four equipped with vibration sensor module static level; In the process of experiment, randomly join some simulated interference vibration data.1 static level, for example, do not make after vibration recognition and identification of sample comparison, as shown in Figure 5.

\section{CONCLUSION}

Through the above two experiments from table 1 and figure 9 shows that the original monitoring equipment acquisition accuracy obviously after temperature compensation than before compensation sampling precision is high, show that in the temperature range under the disturbance of the external environment, and the temperature sensor module will greatly improve the sampling accuracy of monitoring equipment. Also, join the vibration sensor module on monitoring equipment can effectively identify and external interference shielding, cut off is conducive to the emergence of the strange point and improve the sampling accuracy of monitoring equipment, making the next step of analysis, evaluation and prediction steps to provide a scientific and reasonable data.

All of the pros and cons, in combination with the feasibility of the improved scheme of the system and cost range. Not do more harm than good, so the improved version of the settlement of the automated monitoring system with high value of practical use.

\section{ACKNOWLEDGEMENTS}

Foundation item: Study on lightweight of a commercial vehicle frame based on modal experiment (ycjj2016086)

\section{REFERENCES}

[1] Quantico unity, cheung chi-kong. Automatic monitoring system in the application of a high speed rail operation monitoring [J]. Railway survey,2013, pp.1-5.

[2] Zhuo one, wang, Mr. Zhang. High speed railway subsidence SMAIS automation monitoring system research and development and application [J]. Journal of railway engineering, 2015 ,pp.10-15.

[3] Chen Gongliang. Automated subsidence monitoring system construction [J]. Journal of land and resources of Shanghai, 2011, pp.96-98.

[4] Liao Shifang, leaves with a pearl. An high speed railway subgrade settlement automation monitoring system as a whole design [J]. Journal of high-speed railway technology, 2013, pp.26 - 30.

[5] Sun Zexin Zhang Shufeng, Mr. Liu. The static level application in the operational phase of the subway tunnel deformation monitoring and analysis [J]. Modern tunnel technology, 2015, pp.203-208.

[6] Zhang Yuzhi yan-liang du, bao-chen sun, etc. Based on the hydraulic measurement without a frantic jumble of high speed railway roadbed settlement deformation monitoring method [J]. Journal of Beijing jiaotong university, 2013, pp.80-85.

[7] ming-kun liu. Beijing land subsidence monitoring system and the technical method [J]. Geology and resources,2012, pp.245-249.

[8] Evergreen.the static level automatic monitoring system of research [J]. Geology, 2014, pp.30-32.

[9] XiaoJun.Railway roadbed settlement monitoring system and settlement prediction [J]. Construction of Shanxi Province,2014, pp.142-143.

[10] XingTieZeng. Settlement of automatic monitoring system is introduced [J]. Geology,2007, pp.18-19. 